\title{
Therapeutic efficacy of hydrogen-rich saline alone and in combination with PI3K inhibitor in non-small cell lung cancer
}

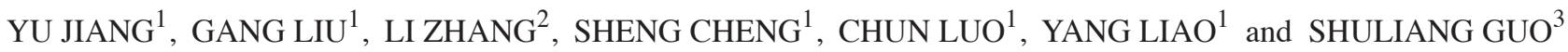 \\ ${ }^{1}$ Department of Respiratory Medicine, The University-Town Hospital Affiliated to Chongqing Medical University, \\ Chongqing 401331; ${ }^{2}$ Department of Pathophysiology, Chongqing Medical University; ${ }^{3}$ Department of Respiratory and \\ Critical Care Medicine, The First Affiliated Hospital of Chongqing Medical University, Chongqing 400016, P.R. China
}

Received October 17, 2017; Accepted June 7, 2018

DOI: $10.3892 / \mathrm{mmr} .2018 .9168$

\begin{abstract}
The aim of the present study was to investigate the effects of combination therapy of LY294002, a specific inhibitor of phosphatidylinositol 3-kinase (PI3K), with hydrogen-rich saline on the proliferation and apoptosis of the non-small cell lung cancer (NSCLC) A549 cell line and the mechanisms underpinning this. Excessive production of reactive oxygen species (ROS) may induce DNA mutations, DNA damage, genomic instability and cell proliferation, and ROS are involved in several types of cancer, particularly lung cancer. In a previous study, hydrogen was recognized as an antioxidant in preventive and therapeutic applications. The $\mathrm{PI} 3 \mathrm{~K} /$ protein kinase B (Akt) pathway is an important signaling pathway that may activate downstream of a series of extracellular signals and impact on cellular processes including cell proliferation, apoptosis and survival. To date, the PI3K/Akt signaling pathway has been indicated as a feasible target for novel antineoplastic drugs. Different strategies combining the two treatment modalities have been used in cancer therapy in order to achieve an improved therapeutic response and longer control of tumor modalities control. The present study investigated the effect of hydrogen-rich saline alone and in combination with the PI3K inhibitor, LY294002, on the proliferation, oxidative stress and apoptosis of NSCLC A549 cells. This combination therapy may be more effective than separate drug treatment; it decreased the malondialdehyde level and increased the superoxide dismutase activity. The combination therapy also enhanced the efficacy of anti-proliferation and apoptosis. Similarly, the results of the present study demonstrated that administration of the two agents in combination may inhibit phospho-Akt activity, and reduce expression of heme oxygenase-1 and nuclear factor- $\kappa \mathrm{B}$ p65. The results
\end{abstract}

Correspondence to: Dr Shuliang Guo, Department of Respiratory and Critical Care Medicine, The First Affiliated Hospital of Chongqing Medical University, Chongqing 400016, P.R. China E-mail: guos1999@yeah.net

Key words: hydrogen-rich saline, phosphoinositide 3-kinase/protein kinase B, reactive oxygen species, lung cancer further suggested that the combination therapy may reduce cell proliferation and promote cell apoptosis by downregulating Akt phosphorylation and inhibiting the PI3K pathway in NSCLC cell lines. Therefore, the present study provided evidence that combined therapy may be a novel therapeutic option for patients with NSCLC.

\section{Introduction}

Lung cancer is the leading cause of cancer death worldwide (1), including China (2). Non-small cell lung cancer (NSCLC) accounts for $>80 \%$ of lung cancer cases. Despite advances in diagnosis and treatment, outcomes remain poor, with the 5 -year survival rate generally being less than $15 \%$ (3). In order to improve the survival rate, numerous studies have aimed to identify novel antineoplastic therapies that have a wide range of biological activities, fewer side effects and low toxicity (2-4).

Oxidative stress is defined as an imbalance between the antioxidant defenses and the production of reactive oxygen species (ROS). It occurs when excessive production of ROS overwhelms their elimination by protective antioxidants or when there is a prominent decrease or lack of antioxidant defense activity (5). ROS are involved in a number of types of cancer (6). Additionally, oxidative stress may affect cancer initiation and progression through increasing DNA mutations or DNA damage, genomic instability and cell proliferation $(6,7)$.

The lungs are directly exposed to higher concentrations of oxygen than other tissues and organs (8). Certain studies have demonstrated that the levels of oxidative stress increase in advanced stages of lung cancer, while the levels of antioxidant molecules decrease (9-11). In particular, patients with squamous cell carcinoma exhibit much higher oxidative stress and ROS, which may be linked to the fact that squamous cell carcinoma is associated with tobacco use $(9,12)$. Enhanced ROS/RNS in the lung may increase the risk to lung cancer through recurring DNA damage, inhibition of apoptosis, and activation of proto-oncogenes by initiating signal transduction pathways (13). The phosphoinositide 3-kinase (PI3K)/protein kinase $\mathrm{B}(\mathrm{Akt})$ pathway is one of most important survival signaling pathways. It has been considered as the predominant growth-factor-activated pathway that may be activated 
downstream of a variety of extracellular signals and that impacts various aspects of cellular processes, including cell proliferation, apoptosis and survival (14). Therefore, numerous types of cancer have been linked to PI3K activity, including lung cancer (15), breast cancer (16), leukemia (17) and melanoma (18), among others $(19,20)$. Therefore, this pathway presents a challenge and an opportunity for cancer therapy. A recent study demonstrated that the PI3K/Akt pathway may be a novel target in antineoplastic therapy (19). However, in a number of cases, activation of the PI3K/Akt pathway alone is not responsible for oncogenic transformation and therefore, the antineoplastic effect of PI3K/mechanistic target of rapamycin (mTOR) inhibitor is not satisfied. In addition, cancer cells resistant to $\mathrm{PI} 3 \mathrm{~K}$ inhibition remain a problem.

Hydrogen is an efficient antioxidant and has been identified as a novel therapeutic antioxidant recently, as it was revealed to selectively reduce cytotoxic ROS in tissues (21-23). In in vivo and in vitro studies, $\mathrm{H} 2$ has exhibited protective antioxidant, anti-apoptotic and anti-inflammatory properties. Hydrogen is safe and effective for distribution into the cytoplasm without the need for specific receptors to overcome hydrophilicity $(24,25)$. In rat models, inhaled hydrogen gas is able to reduce myocardial ischemia reperfusion injury and the infarct size of focal cerebral (26). Due to the fact that hydrogen gas is flammable and inconvenient for clinical use, hydrogen-rich saline with a therapeutic concentration of hydrogen is an alternative model of molecular hydrogen. The protective effect of hydrogen-rich saline is largely due to its ability to reduce the ROS-associated pathologies.

Clinically, combination therapy is most widely used in the treatment of cancer, including lung cancer. The main aim is to achieve synergistic therapeutic effect, to reduce dose and cytotoxicity, and to delay or minimize the induction of drug resistance $(27,28)$.

In the present study, we hypothesized that the combination of hydrogen-rich saline and a highly potent PI3K inhibitor, LY294002, would decrease the proliferation of cancer cells more than single-agent therapy through anti-oxidation and regulation of apoptosis. The present study aimed to demonstrate the effect of the release of inflammatory and apoptosis cytokines. Furthermore, it focused on the influence of combination therapy on the PI3K/Akt signaling pathway and the mechanism underpinning this, which may be applied to clinical tumor therapy.

\section{Materials and methods}

Reagents. Assay kits for superoxide dismutase (SOD) and malondialdehyde (MDA) were purchased from Nanjing Jiancheng Bioengineering Institute (Nanjing, China). A propidium iodide (PI) double staining kit and Annexin V-fluorescein isothiocyanate (FITC) were purchased from Nanjing KeyGene Biotech Co., Ltd. (Nanjing, China). PI was obtained from Sigma-Aldrich (Merck KGaA, Darmstadt, Germany). LY-294002 was purchased from Sigma-Aldrich (Merck KGaA), Stock solution (50 mM) of LY-294002 was dissolved in DMSO and further diluted to a final concentration of $20 \mu \mathrm{M}$. Antibodies were obtained from Santa Cruz Biotechnology, Inc., (Dallas, TX, USA). Other reagents were obtained locally.
Cell lines and cell culture. The human NSCLC A549 cell line was obtained from the Type Culture Collection of the Chinese Academy of Sciences (Shanghai, China). The A549 cell line was maintained in RPMI-1640 medium supplemented with $10 \%$ fetal bovine serum and penicillin/streptomycin at $37^{\circ} \mathrm{C}$ in an atmosphere of $5 \% \mathrm{CO}_{2}$ and $95 \%$ air.

Hydrogen saline. Molecular hydrogen (H2) was dissolved in saline under high pressure $(0.6 \mathrm{MPa})$ to a supersaturated level for $2 \mathrm{~h}$. Hydrogen-rich saline was freshly prepared each week and was sterilized by g radiation. It was ensured that hydrogen-rich saline was maintained at a concentration of more than $0.6 \mathrm{mmol} / \mathrm{l}$. The hydrogen concentration was detected by gas chromatography using the method previously described by Ohsawa et al (22).

Cell proliferation inhibition assay. The inhibition rate of cell proliferation was tested using an MTT assay. A549 cells were grown in 96-well plates at a density of $5 \times 10^{3}$ cells per well for $24 \mathrm{~h}$, prior to being treated with different concentrations of LY294002 (primary concentration, $20 \mu \mathrm{M}$, the concentration of each well is $1 / 3$ of the previous well), hydrogen-rich saline (with a hydrogen concentration of $0.6 \mathrm{mmol} / \mathrm{l}$ ) or a combination of hydrogen-rich saline and LY294002. After $48 \mathrm{~h}$, $20 \mu \mathrm{l} \mathrm{MTT}$ solution [2 $\mathrm{mg} / \mathrm{ml}$ in phosphate-buffered saline (PBS)] was added into each well and the cells were incubated for an additional $4 \mathrm{~h}$ at $37^{\circ} \mathrm{C}$. The medium was completely removed and then $150 \mu \mathrm{l}$ DMSO was added to solubilize the MTT formazan crystals. Finally, the optical density was read at $570 \mathrm{~nm}$ (OD570) using a Microplate reader (Model 550; Bio-Rad Laboratories, Inc., Hercules, MA, USA). Results were presented as the mean of 3 independent experiments each conducted in duplicate. The half-maximal inhibitory concentration (IC50) values were calculated from dose-response curves utilizing GraphPad Prism 6 (GraphPad Software, Inc., La Jolla, CA, USA).

SOD and MDA measurement. A549 cells were grown in 6 -well plates at a density of $1 \times 10^{6}$ cells per well for $24 \mathrm{~h}$, prior to being treated with hydrogen-rich saline $(0.6 \mathrm{mmol} / \mathrm{l})$, LY294002 $(20 \mu \mathrm{M})$ or a combination of hydrogen-rich saline and LY294002 for $24 \mathrm{~h}$. The cells were subsequently harvested, disrupted ultrasonically on ice and centrifuged at 2,500 $\mathrm{x}$ g for $10 \mathrm{~min}$ at $4^{\circ} \mathrm{C}$. The supernatants were collected and stored at $-20^{\circ} \mathrm{C}$ for subsequent analysis. The concentrations of MDA and SOD were evaluated using an MDA and SOD detection kit according to the manufacturer's protocols.

Annexin V/PI flow cytometry analysis. Cell apoptosis was measured using an Annexin V-FITC Apoptosis kit, according to the manufacturer's protocols. A549 cells were grown in 6 -well plates at a density of $1 \times 10^{6}$ cells per well for $24 \mathrm{~h}$, prior to being treated with hydrogen-rich saline $(0.6 \mathrm{mmol} / \mathrm{l})$, LY294002 $(20 \mu \mathrm{M})$ or a combination of hydrogen-rich saline and LY294002 for 24 h. Following staining, flow cytometry was performed using a FACScan flow cytometer. Cells stained as Annexin V-positive and PI-negative were considered apoptotic, while cells that double stained as Annexin V-positive and PI-positive were considered late apoptotic or necrotic. Data were analyzed using the Cell Quest Software 
Program (FACSCalibur ${ }^{\mathrm{TM}}$; BD Biosciences, Franklin Lakes, NJ, USA).

Western blot analysis. The cells were washed twice with ice-cold PBS following treatment for $24 \mathrm{~h}$. Total protein was extracted using cell lysis buffer ( $1 \mathrm{M}$ Tris.HCL pH 7.5, 8M Urea, $150 \mathrm{mM} \beta-\mathrm{ME}$ and $1 \%$ protease inhibitor cocktail). Protein concentration was determined by bicinchoninic acid (BCA) protein assay. Equal amounts of protein $(50 \mu \mathrm{g})$ from each sample were separated by SDS-PAGE. Proteins were subsequently transferred onto polyvinylidene difluoride membranes. Next, the membranes were blocked with 5\% skimmed milk for half an hour in room temperature, prior to being incubated with primary antibodies against HO-1, p65 and p-Akt separately overnight at $4^{\circ} \mathrm{C}$, followed by being washed three times with PBS. The membranes were subsequently incubated with secondary antibodies for $2 \mathrm{~h}$ in room temperature. Following washing, the membranes were visualized with an ECL kit and quantified using ImageJ Software (National Institutes of Health, Bethesda, MD, USA). Experiments were repeated at least three times.

Reverse transcription-quantitative polymerase chain reaction ( $R T-q P C R)$. Total RNA was extracted from cells using an mirVana miRNA Isolation kit (Qiagen $\mathrm{GmbH}$, Hilden, Germany). Equal amounts of RNA samples were reverse transcribed into cDNA using a high-capacity cDNA Reverse Transcription kit (Applied Biosystems; Thermo Fisher Scientific, Inc., Waltham, MA, USA). RT-qPCR was performed using first-strand cDNA with TaqMan probes and TaqMan Universal PCR Master mix (Roche Diagnostics, Basel, Switzerland) with the following conditions: Denaturation at $95^{\circ} \mathrm{C}$ for $10 \mathrm{sec}$, annealing at $58^{\circ} \mathrm{C}$ for $20 \mathrm{sec}$ and elongation at $72^{\circ} \mathrm{C}$ for $20 \mathrm{sec}$, and GAPDH served as an internal control. PCR was performed using the following primers: HO-1 forward, 5'-GATAGAGCGCAACAAGCAGAA-3' and reverse, 5'-CAG TGAGGCCCATACCAGAAG-3'; P65 forward, 5'-GGGAAG GAACGCTGTCAGAG-3' and reverse, 5'-TAGCCTCAGGGT ACTCCATCA-3'; and GAPDH forward, 5'-TGTTGCCAT CAATGACCCCTT-3' and reverse, 5'-CTCCACGACGTA CTCAGCG-3'. Experiments were repeated at least three times. The relative mRNA expression was measured using the $\Delta \Delta \mathrm{Cq}$ method (29).

Statistical analysis. Statistical analyses were performed using GraphPad Prism 6 (GraphPad Software, Inc.). Data are presented as the mean \pm standard error of the mean. The statistical significance of differences between groups in vitro experiments was determined by the Student t-test. The statistical significance of differences between combinations with theoretical combination in vitro experiments was determined by one-way analysis of variance followed by a Turkey's post-hoc test. $\mathrm{P}<0.05$ was considered to indicate a statistically significant difference.

\section{Results}

Inhibition of cell proliferation by hydrogen-rich saline and the PI3K inhibitor, LY294002, in A549 cells. To investigate the proliferation inhibition effects of hydrogen-rich saline alone and in combination with LY-294002 in A549 cells, the cells were treated with hydrogen-rich saline, LY-294002 or a combination of the two for $48 \mathrm{~h}$, and the inhibition of cell proliferation rate was measured using an MTT assay. We observed that growth of A549 cells was suppressed in treatment group (Fig. 1A). Cell proliferation was suppressed following treatment with either hydrogen-rich saline or LY-294002 alone (Fig. 1B). However, compared with treatment with hydrogen-rich saline or LY-294002 alone (Fig. 1C), the combination of the two resulted in increased inhibition.

Effect of hydrogen-rich saline and the PI3K inhibitor, LY294002, on the concentrations of ROS and MDA in A549 cells. To further investigate the effect of a combination of hydrogen-rich saline and the PI3K inhibitor, LY294002, on intracellular oxidant generation in A549 cells, the concentrations of ROS and MDA were examined. The results indicated that the combination of hydrogen-rich saline and LY294002 increased the concentrations of SOD (Fig. 2A), and markedly decreased the levels of MDA, compared with the control group and the hydrogen-rich saline monotherapy group (Fig. 2B). These results demonstrated that the combination of the two treatments suppressed intracellular oxidant generation in A549 cells.

Induction of apoptosis by hydrogen-rich saline and the PI3K inhibitor, LY294002, in A549 cells. A549 cells were treated with hydrogen saline, the PI3K inhibitor, LY294002, or the combination of hydrogen saline with LY294002 for $24 \mathrm{~h}$, prior to being analyzed by flow cytometry. In comparison with the control group, the numbers of early and late apoptotic cells were markedly increased (Fig. 3A and B). The proportion of early and late apoptotic cells in the combination treatment group was markedly increased, compared with the control group. The proportion of apoptotic cells was increased in the three groups, but the combination of the two treatments induced a more marked increase than treatment with hydrogen-rich saline or LY-294002 alone.

Inhibition of $\mathrm{HO}-1$ and $N F-\kappa B$ p 65 protein and $m R N A$ expression in A549 cells by combination of hydrogen-rich saline and the PI3K inhibitor, LY294002. To investigate the mechanism of anti-oxidation induced by hydrogen-rich saline alone, LY294002 alone and the combination of hydrogen-rich saline and LY294002, the present study tested the effect of three treatment groups on HO-1 and NF- $\kappa \mathrm{B}$ p 65 protein and mRNA expression levels. Western blot analysis revealed that hydrogen-rich saline monotherapy led to a decrease in $\mathrm{HO}-1$ and p65 levels as compared with the control cells (Fig. 4A and B). RT-qPCR analysis revealed that hydrogen-rich saline monotherapy led to a decrease in HO- 1 and p65 mRNA expression levels, compared with the control cells (Fig. 5A and B). The protein and mRNA expression levels were higher following combined treatment with hydrogen-rich saline and LY294002, compared with treatment with either of the agents alone.

Inhibition of the PI3K/Akt pathway by hydrogen-rich saline alone and in combination with LY294002. In order to better understand the molecular basis of the anti-proliferation and apoptosis induced by hydrogen-rich saline alone and in 


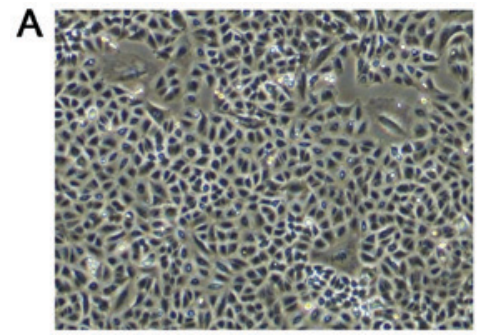

Control

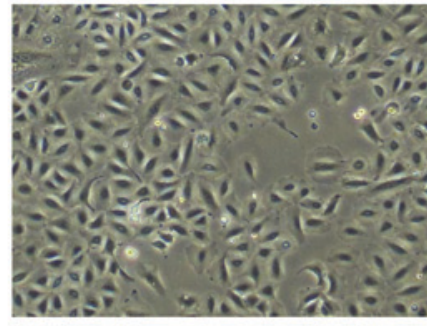

HYDROGEN

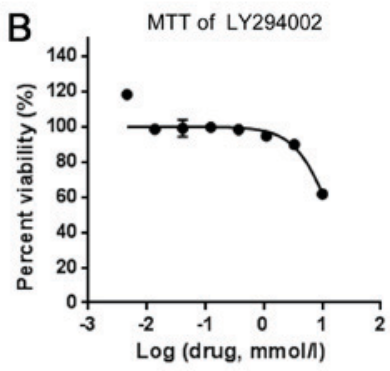

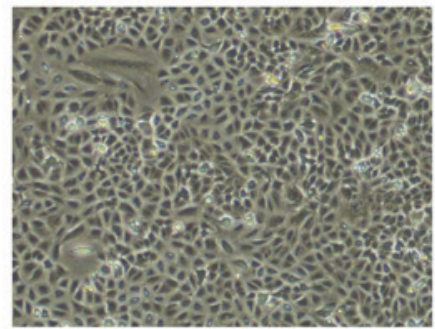

LY294002

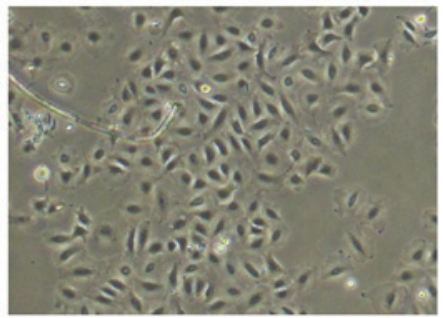

HYDROGEN+LY294002

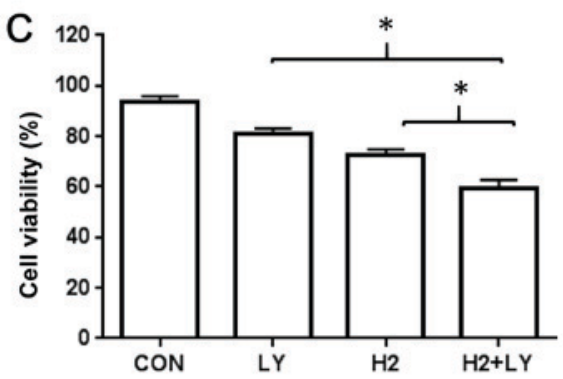

Figure 1. Effects of hydrogen-rich saline and in combination with the PI3K inhibitor LY294002 on cell proliferation in A549 cells. (A) NSCLC cell line (A549) treated with hydrogen-rich saline, LY294002 and combination was incubated for $24 \mathrm{~h}$ (magnification, x100). (B) Percentage viability of the cells was determined after cells were incubated with MTT, and (C) with the different groups. Optical density measurements were made at 570 nm. Data are presented as the mean \pm standard deviation for 3 independent experiments. ${ }^{*} \mathrm{P}<0.05$, as indicated. PI3K, phosphatidylinositol 3-kinase; NSCLC, non-small cell lung cancer; CON, control; LY, LY294002; H2, molecular hydrogen.
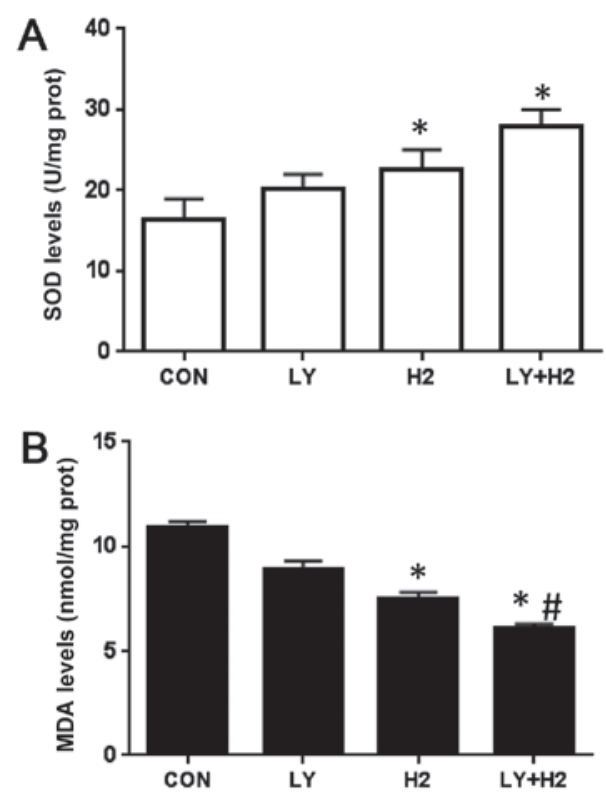

Figure 2. Effects of hydrogen-rich saline and in combination with PI3K inhibitor LY294002 on intracellular oxidant generation and antioxidant enzyme activity in A549 cells. (A) SOD and (B) MDA levels were measured using a microplate spectrophotometer. Data are presented as the mean \pm standard deviation for 3 independent experiments. ${ }^{*} \mathrm{P}<0.05$ vs. $\mathrm{CON}$; ${ }^{\text {}} \mathrm{P}<0.05$ vs. H2. PI3K, phosphatidylinositol 3-kinase; CON, control; LY, LY294002; H2, molecular hydrogen; SOD, superoxide dismutase; MDA, malondialdehyde. combination with LY294002, the present study examined the expression of p-Akt, an effector of the PI3K/Akt/mTOR signaling pathway, following treatment with hydrogen-rich saline alone and in combination with LY294002 for $24 \mathrm{~h}$. The protein expression of p-Akt was decreased in response to hydrogen-rich saline alone, LY294002 alone and a combination of the two, while the p-Akt protein expression level remained lower in the combined treatment group than following drug treatment alone (Fig. 6A and B). These results indicated that the combination therapy has a synergistic effect on anti-proliferation and the induction of apoptosis possibly through inhibiting the PI3K/Akt pathway.

\section{Discussion}

To the best of our knowledge, oxidative stress play a key role in tumorigenesis $(6,30-32)$. Oxidative stress may affect the initiation and progression of cancer by leading to DNA mutations, DNA damage, inducing genomic instability and cell proliferation (33-36).

In particular, oxidative damage serves a pivotal role in pulmonary disease as the lungs are exposed daily to oxidants, which are generated either endogenously or exogenously (e.g., air pollutants and cigarette smoke) (10); tobacco smoke and particulate matter in air pollution may generate ROS and therefore increase the incidence of lung cancer and 

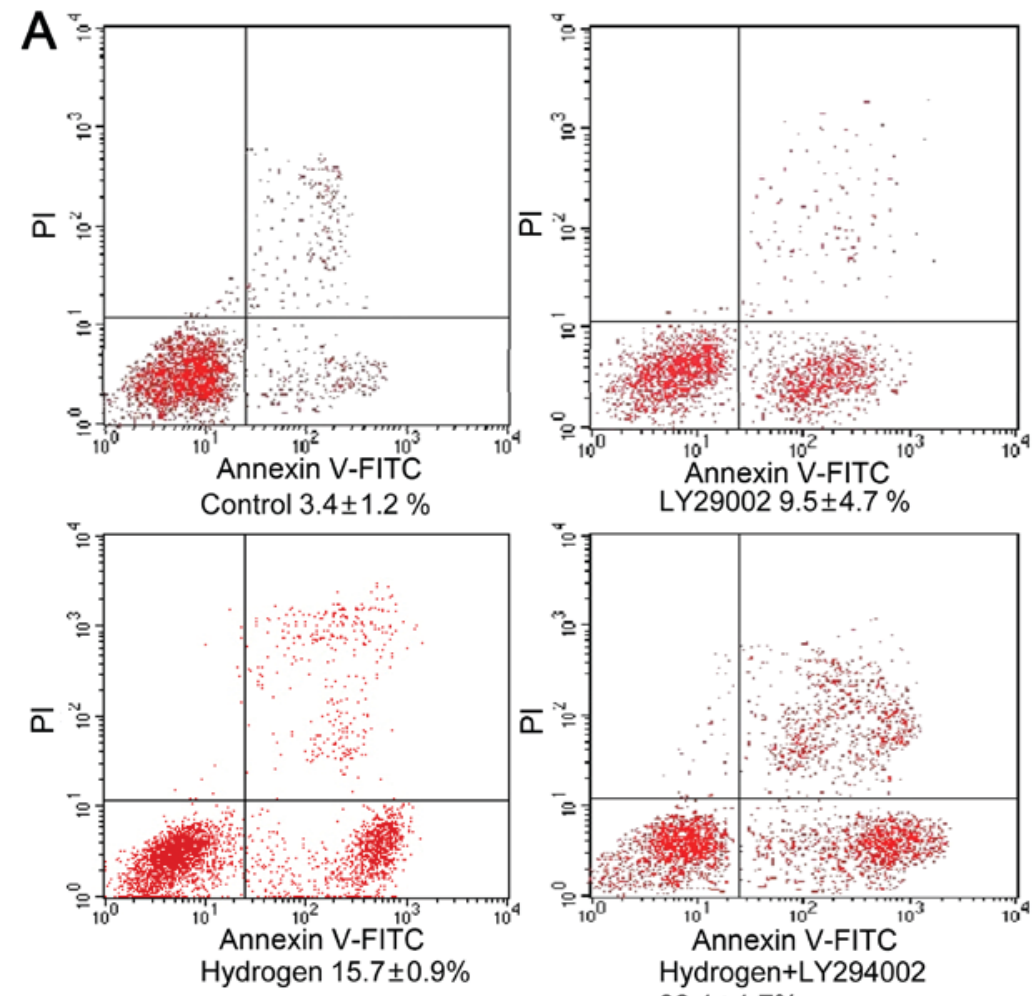

B

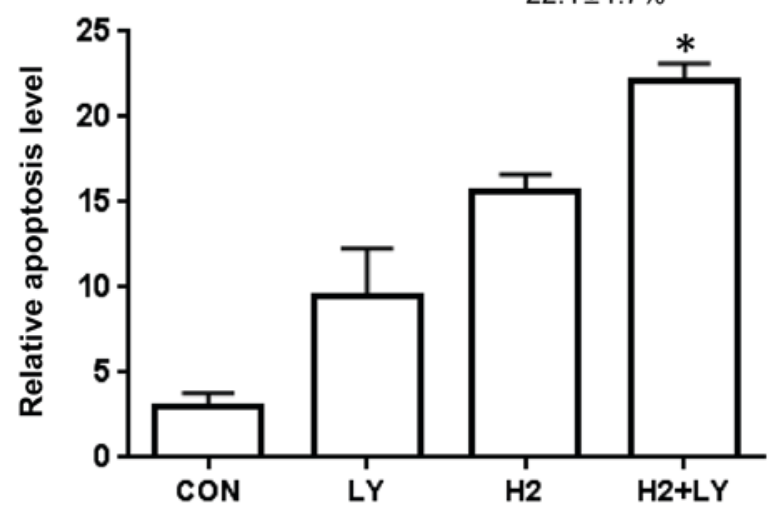

Figure 3. Effects of hydrogen-rich saline and LY294002 as single-agents and in combination on the survival of A549 cell line. A549 non-small-cell lung cancer cells were treated with hydrogen-rich saline $(0.6 \mathrm{mmol} / \mathrm{l})$ and LY294002 $(1 \mu \mathrm{mol} / \mathrm{l})$, each alone or in combination, for $24 \mathrm{~h}$. (A) Single drug or combination-induced apoptosis as analyzed by Annexin V-FITC and PI staining and flow cytometry. (B) The percentage of apoptotic cells was determined by Annexin V and PI staining. Data are presented as the mean \pm standard deviation for 3 independent experiments. "P<0.05 vs. single drug. CON, control; LY, LY294002; H2, molecular hydrogen; FTIC, fluorescein isothiocyanate; PI, propidium iodide.

respiratory diseases (37). Furthermore, synergistic effects in the generation of ROS contribute toward lung cancer through inducing oxidative stress and inflammation with a high DNA damage potential (10). Since oxidative stress is a crucial event in cancer cells, this may also provide an opportunity to kill malignant cells.

Hydrogen has been generally recognized as a therapeutic anti-oxidative and anti-apoptotic tool $(22,38)$. Numerous studies have demonstrated that hydrogen may selectively react with exclusively detrimental ROS, while hydrogen does not disturb physiological metabolic oxidation-reduction reactions or disrupt the ROS involved in cell signaling $(22,31,39)$. Hydrogen-rich saline, which is easily administered and is safe for clinical application, is a viable approach with the same properties of anti-oxidation, anti-inflammation and anti-apoptosis, and has been used in several recent animal disease studies (40-42). However, a study has demonstrated that hydrogen therapy may inhibit the proliferation of tongue carcinoma cells (43) and reduce the size of skin tumors. Further studies will elucidate the molecular mechanism of hydrogen-mediated inhibition of cancer cell proliferation.

The present study initially applied hydrogen-rich saline alone to lung cancer cells. Subsequently, the effect of hydrogen-rich saline on apoptosis and inflammatory cytokines, and the pathway involved in this process, was investigated. The results demonstrated the following: i) Hydrogen-rich saline treatment alone inhibited A549 cell proliferation; ii) hydrogen-rich saline treatment alone decreased MDA expression and increased SOD activity; iii) hydrogen-rich saline treatment alone induced A549 cell apoptosis; iv) in in vitro experiment, treatment with hydrogen significantly suppressed the effect on protein and mRNA expression of 
A
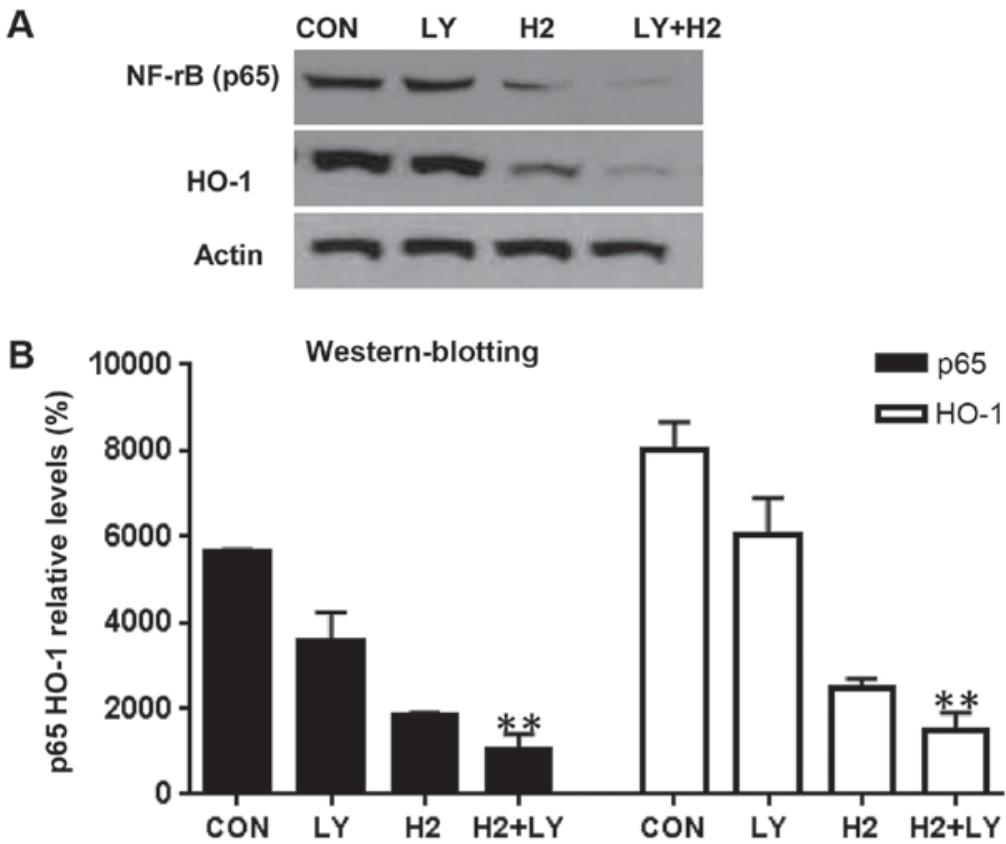

Figure 4. Western blotting analysis of A549 cells treated as indicated for $24 \mathrm{~h}$. (A) Western blot results of the protein expression levels of HO-1 and NF- $\mathrm{kB}$ p65 in A549 cells treated with single agent and combination. $\beta$-actin was used as a loading control. Representative images from three independent experiments are shown. (B) Treatment with hydrogen-rich saline in combination with LY294002 led to a significant decrease in HO-1 and NF- $\mathrm{kB}$ p65. Data are presented as the mean \pm standard deviation for 3 independent experiments. ${ }^{* *} \mathrm{P}<0.01$ vs. control. CON, control; LY, LY294002; H2, molecular hydrogen; HO-1, heme oxygenase; NF-, nuclear factor.
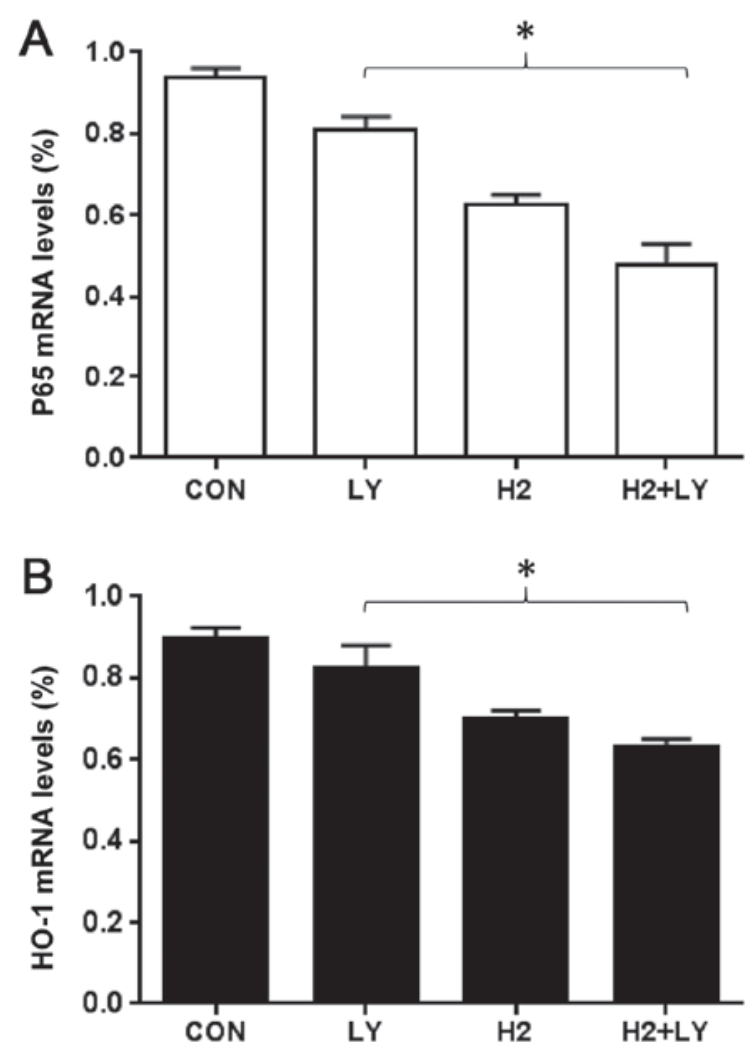

Figure 5. (A and B) Reverse transcription-quantitative polymerase chain reaction analysis of A549 cells treated as indicated. The mRNA expression of NF- $\mathrm{kB}$ p65 and HO-1 following treatment with hydrogen-rich saline, LY294002 alone and combination hydrogen-rich saline with LY294002 on A549 cells. Note that treatment with hydrogen-rich saline in combination with LY294002 led to a significant decrease in the mRNA expression of HO-1 and $\mathrm{p} 65$. Data are presented as the mean \pm standard deviation for 3 independent experiments. " $\mathrm{P}<0.05$, as indicated. CON, control; LY, LY294002; H2, molecular hydrogen; HO-1, heme oxygenase; NF-, nuclear factor.
HO-1 and NF- $\kappa$ B p65 in A549 cell and v) hydrogen-rich saline suppressed the expression of p-Akt and the expression levels of HO-1 and $\mathrm{p} 65$.

The results of the present study demonstrated that hydrogen-rich saline alone may downregulate the expression of NF- $\kappa \mathrm{B}$ p65, which is a redox-regulated transcription factor. The activation of $\mathrm{NF}-\kappa \mathrm{B}$ serves an important role in regulating the expression of a number of early stress response genes, which are associated with oxidative stress-induced cell death in a wide range of tumor types (44). Therefore, it is suggested that hydrogen-rich saline may attenuate oxidative stress by inhibiting the activation of $\mathrm{NF}-\kappa \mathrm{B}$. The present study demonstrated that hydrogen-rich saline alone downregulated the expression of HO-1, which is a stress response protein (45). As HO-1 expression and activity are markedly increased by stressful conditions, the major role of $\mathrm{HO}-1$ is involved in antioxidant and anti-inflammatory responses $(46,47)$. However, the induction of $\mathrm{HO}-1$, which may provide cells with a growth advantage, is not always adequate to protect the cells. HO-1 may be overexpressed in tumor cells $(45,48,49)$. In the present study, HO-1 was downregulated in vitro, oxidative stress was markedly decreased by hydrogen-rich saline and the expression of p-Akt was suppressed. These results indicated that it is possible that the anti-proliferation and apoptosis inducing functions of hydrogen-rich saline are primarily achieved by downregulating transcriptional factors $(\mathrm{NF}-\kappa \mathrm{B}$ and $\mathrm{p}-\mathrm{Akt}) \mathrm{via}$ the PI3K/Akt pathway.

The PI3K/Akt pathway is involved crucially in the development and progression of several types of cancer by aiding in promoting cell proliferation and allowing cells to evade apoptosis. PI3K/Akt pathway activation is associated with poor outcomes in certain types of cancer (50). In addition, numerous novel 'targeted agents' have been specifically exploited to act 

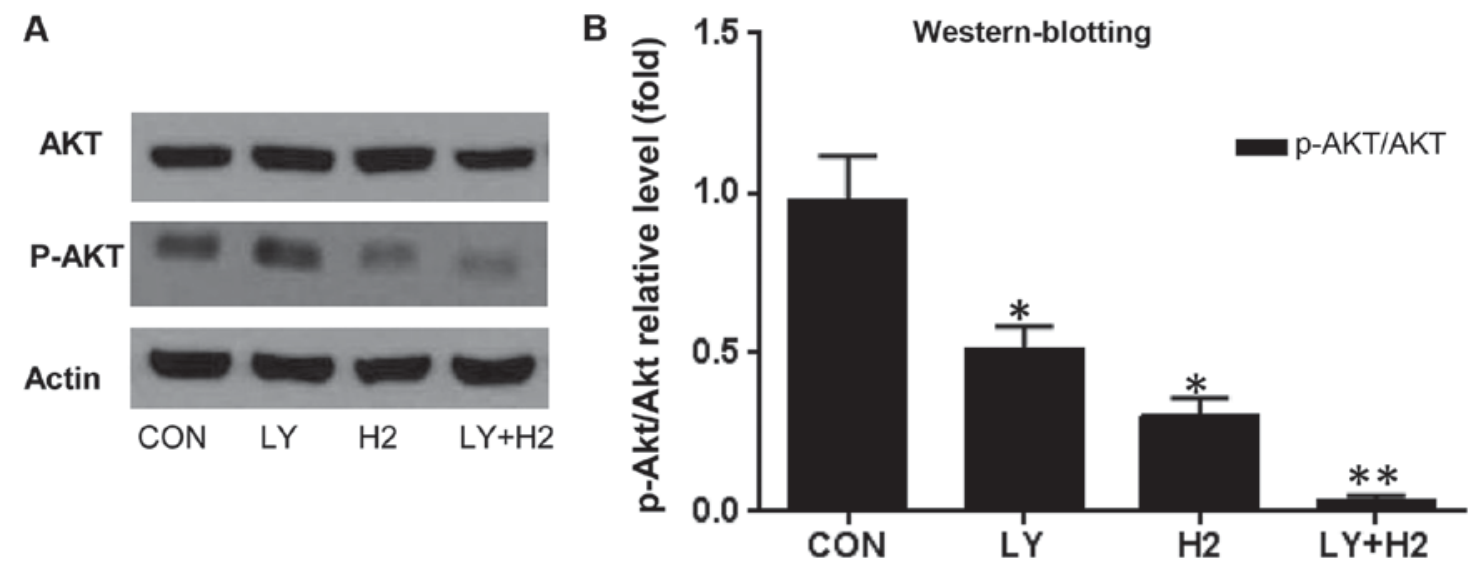

Figure 6. (A and B) Effects of hydrogen-rich saline and LY294002 as single-agents and in combination on PI3K/p-AKT pathway of NSCLC cells. (A) Western blot analysis of proteins (pAKT and AKT) was indicated in A549 cells treated with hydrogen-rich saline and LY294002 as single agents or in combination for $24 \mathrm{~h}$. Actin was used as a loading control. (B) Treatment with hydrogen-rich saline in combination with LY294002 led to a significant decrease of protein expression of p-Akt/Akt. Data are presented as the mean \pm standard deviation for 3 independent experiments. " $\mathrm{P}<0.05$ and ${ }^{* * *} \mathrm{P}<0.01 \mathrm{vs}$. CON. PI3K, phosphatidylinositol 3-kinase; AKT, protein kinase B; NSCLC, non-small cell lung cancer; CON, control; LY, LY294002; H2, molecular hydrogen; p-, phosphorylated.

on PI3K/Akt and downstream component-related targets due to the ubiquitous nature of the activation of this pathway in cancer. Furthermore, multiple PI3K/Akt pathway inhibitors are now under active clinical development (20).

In NSCLC cells, these small molecule inhibitors have been demonstrated to shift the apoptotic threshold in cancer cells following treatment with other molecule inhibitors or standard chemotherapy (51).

The present study focused on the efficacy of a PI3K inhibitor (LY294002) in combination with anti-oxidative treatment with hydrogen-rich saline. Regarding the administration of hydrogen-rich saline or LY294002 separately as single agents, the results of the present study demonstrated that the combination treatment of A549 cells may be more effective than single treatment. Combination treatment enhanced the efficacy of anti-proliferation and apoptosis induction. Similarly, these data demonstrated that usage of the two agents in combination may decrease MDA expression levels, inhibit p-Akt activity, and reduce expression of $\mathrm{HO}-1$ and $\mathrm{NF}-\kappa \mathrm{B}$. The results of the present study supported the hypothesis that the combination therapy has the effect of anti-proliferation and apoptosis induction through inhibiting the activation of Akt phosphorylation and reducing the downstream expression of HO-1 and NF- $\kappa$ B. Therefore, combining PI3K inhibitor (LY294002) and hydrogen-rich saline may negatively regulate A549 cell proliferation through inhibition of the PI3K/Akt pathway. The present study has suggested that this combination is able to serve as dual PI3K pathway inhibitors, which may be attractive targets for the treatment of lung cancer, with a low risk of side effects. However, due to the fact that the present study using combination are unable to assay clear mechanisms of PI3K in the cells, clarification of the intracellular signaling network of cells should be verified in future experiments.

In conclusion, the present study demonstrated that hydrogen-rich saline in combination with LY294002 treatment in A549 cancer cells leads to constitutive inhibition of the PI3K/Akt signaling pathway and acts as a negative regulator for cell proliferation and expression of HO-1. Furthermore, the drug combination approach used in the present study may be useful for the study of novel cancer therapeutics and may directly aid future drug development and patient stratification in clinical trials.

\section{Acknowledgements}

Not applicable.

\section{Funding}

The present study was supported by the grant from the Medical Research Projects of Chongqing Municipal Health and Family Planning Commission (grant no. 2016MSXM057).

\section{Availability of data and materials}

The datasets used and/or analyzed during the current study are available from the corresponding author on reasonable request.

\section{Authors' contributions}

YJ and SLG planned the experiments. GL, SC and CL performed the experiments. LZ and YL analyzed the data. YJ prepared the figures. YJ and LZ drafted the manuscript. YJ and LZ proof read and approved the final version of the manuscript.

\section{Ethics approval and consent to participate}

Not applicable.

\section{Patient consent for publication}

Not applicable.

\section{Competing interests}

The authors declare that they have no competing interests. 


\section{References}

1. Siegel RL, Miller KD and Jemal A: Cancer statistics, 2017. CA Cancer J Clin 67: 7-30, 2017.

2. Chen W, Zheng R, Baade PD, Zhang S, Zeng H, Bray F, Jemal A, Yu XQ and He J: Cancer statistics in China, 2015. CA Cancer J Clin 66: 115-132, 2016

3. Jemal A, Siegel R, Ward E, Murray T, Xu J and Thun MJ: Cancer statistics, 2007. CA Cancer J Clin 57: 43-66, 2007.

4. Yan Z, Dai Y, Fu H, Zheng Y, Bao D, Yin Y, Chen Q, Nie X, Hao Q, Hou D and Cui Y: Curcumin exerts a protective effect against premature ovarian failure in mice. J Mol Endocrinol 60 : 261-271, 2018.

5. Duracková Z: Some current insights into oxidative stress. Physiol Res 59: 459-469, 2010.

6. Reuter S, Gupta SC, Chaturvedi MM and Aggarwal BB: Oxidative stress, inflammation, and cancer: How are they linked? Free Radic Biol Med 49: 1603-1616, 2010

7. Visconti R and Grieco D: New insights on oxidative stress in cancer. Curr Opin Drug Discov Devel 12: 240-245, 2009

8. Kinnula VL and Crapo JD: Superoxide dismutases in the lung and human lung diseases. Am J Respir Crit Care Med 167: $1600-1619,2003$

9. Esme H, Cemek M, Sezer M, Saglam H, Demir A, Melek H and Unlu M: High levels of oxidative stress in patients with advanced lung cancer. Respirology 13: 112-116, 2008.

10. Valavanidis A, Vlachogianni T, Fiotakis K and Loridas S: Pulmonary oxidative stress, inflammation and cancer: Respirable particulate matter, fibrous dusts and ozone as major causes of lung carcinogenesis through reactive oxygen species mechanisms. Int J Environ Res Public Health 10: 3886-3907, 2013.

11. Møller P, Folkmann JK, Forchhammer L, Bräuner EV, Danielsen PH, Risom L and Loft S: Air pollution, oxidative damage to DNA, and carcinogenesis. Cancer Lett 266: 84-97, 2008.

12. Hawthorn L, Stein L, Panzarella J, Loewen GM and Baumann H: Characterization of cell-type specific profiles in tissues and isolated cells from squamous cell carcinomas of the lung. Lung Cancer 53: 129-142, 2006.

13. Azad N, Rojanasakul Y and Vallyathan V: Inflammation and lung cancer: Roles of reactive oxygen/nitrogen species. J Toxicol Environ Health B Crit Rev 11: 1-15, 2008.

14. Dillon RL, White DE and Muller WJ: The phosphatidyl inositol 3-kinase signaling network: Implications for human breast cancer. Oncogene 26: 1338-1345, 2007.

15. Gustafson AM, Soldi R, Anderlind C, Scholand MB, Qian J, Zhang X, Cooper K, Walker D, McWilliams A, Liu G, et al: Airway PI3K pathway activation is an early and reversible event in lung cancer development. Sci Transl Med 2: 26ra25, 2010.

16. Fry MJ: Phosphoinositide 3-kinase signalling in breast cancer: How big a role might it play? Breast Cancer Res 3: 304-312, 2001

17. Martelli AM, Evangelisti C, Chappell W, Abrams SL, Bäsecke J, Stivala F, Donia M, Fagone P, Nicoletti F, Libra M, et al: Targeting the translational apparatus to improve leukemia therapy: Roles of the PI3K/PTEN/Akt/mTOR pathway. Leukemia 25: 1064-1079, 2011.

18. Davies MA: The role of the PI3K-AKT pathway in melanoma. Cancer J 18: 142-147, 2012

19. Chang F, Lee JT, Navolanic PM, Steelman LS, Shelton JG, Blalock WL, Franklin RA and McCubrey JA: Involvement of PI3K/Akt pathway in cell cycle progression, apoptosis, and neoplastic transformation: A target for cancer chemotherapy. Leukemia 17: 590-603, 2003

20. Wong KK, Engelman JA and Cantley LC: Targeting the PI3K signaling pathway in cancer. Curr Opin Genet Dev 20: 87-90, 2010

21. Nakao A, Toyoda Y, Sharma P, Evans M and Guthrie N: Effectiveness of hydrogen rich water on antioxidant status of subjects with potential metabolic syndrome-an open label pilot study. J Clin Biochem Nutr 46: 140-149, 2010.

22. Ohsawa I, Ishikawa M, Takahashi $K$, Watanabe M, Nishimaki $K$, Yamagata K, Katsura K, Katayama Y, Asoh S and Ohta S: Hydrogen acts as a therapeutic antioxidant by selectively reducing cytotoxic oxygen radicals. Nat Med 13: 688-694, 2007.

23. Buchholz BM, Kaczorowski DJ, Sugimoto R, Yang R, Wang Y, Billiar TR, McCurry KR, Bauer AJ and Nakao A: Hydrogen inhalation ameliorates oxidative stress in transplantation induced intestinal graft injury. Am J Transplant 8: 2015-2024, 2008.

24. Ohta S: Molecular hydrogen is a novel antioxidant to efficiently reduce oxidative stress with potential for the improvement of mitochondrial diseases. Biochim Biophys Acta 1820: 586-594, 2012.
25. Takaki A, Kawai D and Yamamoto K: Multiple hits, including oxidative stress, as pathogenesis and treatment target in non-alcoholic steatohepatitis (NASH). Int J Mol Sci 14: 20704-20728, 2013.

26. Fukuda K, Asoh S, Ishikawa M, Yamamoto Y, Ohsawa I and Ohta S: Inhalation of hydrogen gas suppresses hepatic injury caused by ischemia/reperfusion through reducing oxidative stress. Biochem Biophys Res Commun 361: 670-674, 2007.

27. Chou TC: Theoretical basis, experimental design, and computerized simulation of synergism and antagonism in drug combination studies. Pharmacol Rev 58: 621-681, 2006.

28. Desai UN, Shah KP, Mirza SH, Panchal DK, Parikh SK and Rawal RM: Enhancement of the cytotoxic effects of Cytarabine in synergism with Hesperidine and Silibinin in Acute Myeloid Leukemia: An in-vitro approach. J Cancer Res Ther 11: 352-357, 2015.

29. Livak KJ and Schmittgen TD: Analysis of relative gene expression data using real-time quantitative PCR and the 2(-Delta Delta C(T)) method. Methods 25: 402-408, 2001

30. Barreiro E, Fermoselle C, Mateu-Jimenez M, Sánchez-Font A, Pijuan L, Gea J and Curull V: Oxidative stress and inflammation in the normal airways and blood of patients with lung cancer and COPD. Free Radic Biol Med 65: 859-871, 2013

31. Khansari N, Shakiba Y and Mahmoudi M: Chronic inflammation and oxidative stress as a major cause of age-related diseases and cancer. Recent Pat Inflamm Allergy Drug Discov 3: 73-80, 2009.

32. Glasauer A and Chandel NS: Targeting antioxidants for cancer therapy. Biochem Pharmacol 92: 90-101, 2014.

33. Ames BN and Gold LS: Animal cancer tests and cancer prevention. J Natl Cancer Inst Monogr: 125-132, 1992

34. Guyton KZ and Kensler TW: Oxidative mechanisms in carcinogenesis. Br Med Bull 49: 523-544, 1993.

35. Schulte-Hermann R, Timmermann-Trosiener I, Barthel G and Bursch W: DNA synthesis, apoptosis, and phenotypic expression as determinants of growth of altered foci in rat liver during phenobarbital promotion. Cancer Res 50: 5127-5135, 1990.

36. Klaunig JE, Xu Y, Isenberg JS, Bachowski S, Kolaja KL, Jiang J, Stevenson DE and Walborg EF Jr: The role of oxidative stress in chemical carcinogenesis. Environ Health Perspect 106 (Suppl 1): S289-S295, 1998.

37. Strak M, Janssen NA, Godri KJ, Gosens I, Mudway IS, Cassee FR, Lebret E, Kelly FJ, Harrison RM, Brunekreef B, et al: Respiratory health effects of airborne particulate matter: The role of particle size, composition, and oxidative potential-the RAPTES project. Environ Health Perspect 120: 1183-1189, 2012.

38. Wood KC and Gladwin MT: The hydrogen highway to reperfusion therapy. Nat Med 13: 673-674, 2007.

39. Chen H, Sun YP, Li Y, Liu WW, Xiang HG, Fan LY, Sun Q, Xu XY, Cai JM, Ruan CP, et al: Hydrogen-rich saline ameliorates the severity of 1-arginine-induced acute pancreatitis in rats. Biochem Biophys Res Commun 393: 308-313, 2010

40. Chen HG, Xie KL, Han HZ, Wang WN, Liu DQ, Wang GL, and $\mathrm{Yu} \mathrm{YH}$ : Heme oxygenase-1 mediates the anti-inflammatory effect of molecular hydrogen in LPS-stimulated RAW 264.7 macrophages. Int J Surg 11: 1060-1066, 2013.

41. Xie K, Yu Y, Huang Y, Zheng L, Li J, Chen H, Han H, Hou L, Gong $\mathrm{G}$ and Wang G: Molecular hydrogen ameliorates lipopolysaccharide-induced acute lung injury in mice through reducing inflammation and apoptosis. Shock 37: 548-555, 2012.

42. Kajiyama S, Hasegawa G, Asano M, Hosoda H, Fukui M, Nakamura N, Kitawaki J, Imai S, Nakano K, Ohta M, et al: Supplementation of hydrogen-rich water improves lipid and glucose metabolism in patients with type 2 diabetes or impaired glucose tolerance. Nutr Res 28: 137-143, 2008.

43. Huang CS, Kawamura T, Toyoda Y and Nakao A: Recent advances in hydrogen research as a therapeutic medical gas. Free Radic Res 44: 971-982, 2010.

44. Wang C, Li J, Liu Q, Yang R, Zhang JH, Cao YP and Sun XJ: Hydrogen-rich saline reduces oxidative stress and inflammation by inhibit of JNK and NF- $\kappa$ B activation in a rat model of amyloid-beta-induced Alzheimer's disease. Neurosci Lett 491: 127-132, 2011.

45. Sass G, Leukel P, Schmitz V, Raskopf E, Ocker M, Neureiter D, Meissnitzer M, Tasika E, Tannapfel A and Tiegs G: Inhibition of heme oxygenase 1 expression by small interfering RNA decreases orthotopic tumor growth in livers of mice. Int J Cancer 123: 1269-1277, 2008

46. Nitti M, Piras S, Marinari UM, Moretta L, Pronzato MA and Furfaro AL: HO-1 induction in cancer progression: A matter of cell adaptation. Antioxidants (Basel) 6: pii: E29, 2017. 
47. Lien GS, Wu MS, Bien MY, Chen $\mathrm{CH}$, Lin $\mathrm{CH}$ and Chen $\mathrm{BC}$ Epidermal growth factor stimulates nuclear factor- $\kappa \mathrm{B}$ activation and heme oxygenase-1 expression via c-Src, NADPH oxidase, PI3K, and Akt in human colon cancer cells. PLoS One 9: e104891, 2014.

48. Was H, Dulak J and Jozkowicz A: Heme oxygenase-1 in tumor biology and therapy. Curr Drug Targets 11: 1551-1570, 2010.

49. Goodman AI, Choudhury M, da Silva JL, Schwartzman ML and Abraham NG: Overexpression of the heme oxygenase gene in renal cell carcinoma. Proc Soc Exp Biol Med 214: 54-61, 1997.
50. Engelman JA: Targeting PI3K signalling in cancer: Opportunities, challenges and limitations. Nat Rev Cancer 9: 550-562, 2009.

51. Schmidt M, Hövelmann S and Beckers TL: A novel form of constitutively active farnesylated Akt1 prevents mammary epithelial cells from anoikis and suppresses chemotherapy-induced apoptosis. Br J Cancer 87: 924-932, 2002.

(i) (3) This work is licensed under a Creative Commons Attribution-NonCommercial-NoDerivatives 4.0 International (CC BY-NC-ND 4.0) License. 